\title{
Citations
}

\section{One size fits all}

When designing biosensors, researchers usually face frustrating tradeoffs, e.g. a platform adept at detecting nucleic acids may stumble when it comes to proteins or small molecules. Convergence may be at hand, however, as a recent article in Nature Chemistry describes the development of a universal detector. As their starting point, Das et al. selected an electrochemical detection scheme in which an electron acceptor is attracted to a nanoelectrode surface in proportion to the charge state of a tethered oligonucleotide probe. Because of the negative charge of the aptamer's phosphate backbone, this platform tends to suffer from a high-background signal and a dependence on the analyte triggering a meaningful change in electrostatics-a real headache for small molecules or proteins that are not highly charged. To guarantee a measurable change in charge, Das et al. introduced the neutralizer displacement assay (NDA). Here, the probe's initial charge is masked by a neutralizer, or a partially complementary peptide nucleic acid with five lysine residues at each terminus. This molecule neutralizes the charge at the sensor surface adjacent to the probe; when the analyte of interest displaces the neutralizer, a shift in charge should result. To test their theory, Das et al. investigated the suitability of the NDA for detecting two small molecules, ATP and cocaine. In each case, the current associated with the aptamer probe was substantially decreased in the presence of a neutralizer with two mismatches. When analyte was added, there was a $60-100 \%$ change in current, marking the first time this nanostructured microelectrode platform has been shown capable of detecting small molecules. Importantly, this capability does not come at the expense of nucleic acid detection. Using the NDA with a 20 -mer target sequence, the authors observed concentration-

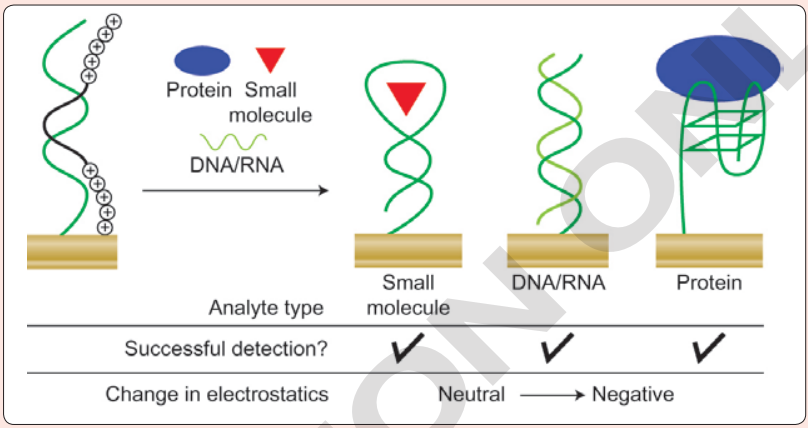

Displacement of a neutralizer oligo for readily detectable electrostatic changes in the neutralizer displacement assay. Source: Nature Chemistry

dependent signal changes over five orders of magnitude, with a detection limit in the range of $100 \mathrm{aM}$. Under less ideal conditions, where matrix effects arise, target-specific signal was still readily detectable. A variation of the technique-in which the neutralizer is complementary to the target-also showed highly specific DNA detection. In tests with a thrombin aptamer, Das et al. confirm the technique is also applicable to protein detection. With appropriate chip design, multiplexing of up to 100 probes should be feasible, suggesting sensitive, simultaneous detection of tens of nucleic acid, protein, and small molecule analytes are within reach.

J. Das et al. An ultrasensitive universal detector based on neutralizer displacement. Nat Chem. [Epub ahead of print, June 3, 2012; doi:10.1038/nchem.1367].

\section{Spy cam}

Incubators are great places to grow cells, but not so helpful when it comes to observing what the cells are up to. In a typical time course experiment, flasks or dishes must be removed periodically for imaging under a microscope. Shuttling between incubator and microscope can be avoided with specialized live-cell imaging chambers or an incubator enclosure surrounding the microscope itself. The technical complexity and expense of these approaches can impose substantial barriers, which makes an article from Kim et al. appearing in Lab on a Chip so welcome. In this approach, the microscope is downsized to the extent that it can fit in a standard tissue culture incubator. Although compact imaging systems have been described and are even commercially available, these devices tend to be lens-free, which imposes strict limits on the distance the target object can be from the imaging system and requires image reconstruction algorithms. By contrast, Kim et al. couldn't make their mini-microscope much simpler: they open up a commercial webcam, reversed the plastic lens (housing it within a cut-off microcentrifuge tube), fashioned a frame to orient the lens to the webcam imaging sensor, and placed it underneath a flask or dish, with an LED for illumination. The mini-microscope fits easily within an incubator and connects through a USB port to a computer, where image capture and processing are handled with a MATLAB program. The mini-microscope can manage $100 \times$ to $400 \times$ magnification, with a resolution of about $1.4 \mu \mathrm{m}$. The image quality is sufficient for monitoring in vitro wound repair via the scratch assay or for gauging embryoid body formation in stem cell biology studies. The mini-microscope is not limited to live-cell monitoring, however, as the authors show that the system can track microdroplet formation in microfluidic chips, an important digital fluidics application. For more demanding uses, Kim et al. recommends swapping the default webcam lens with a higherresolution variant and trading up to a highspeed image sensor. Even in its most basic implementation, however, the mini-microscope offers surprising flexibility and utility in projects for which conventional microscopes are too unwieldy.

Kim et al. A mini-microscope for in situ monitoring of cells. Lab Chip. [Epub ahead of print, July 4, 2012; doi:10.1039/ C2LC40345E].

Selected and written by Nijsje Dorman, Ph.D. [CHA

BioTechniques 53:127 (September 2012)

doi 10.2144/000113917 